\title{
Lanosterol induces mitochondrial uncoupling and protects dopaminergic neurons from cell death in a model for Parkinson's disease
}

\author{
L Lim ${ }^{\star, 1}$, V Jackson-Lewis ${ }^{2}$, LC Wong ${ }^{3}$, GH Shui ${ }^{4}$, AXH Goh ${ }^{4}$, S Kesavapany ${ }^{4}$, AM Jenner,7, M Fivaz ${ }^{3,5}$, S Przedborski ${ }^{2}$ \\ and MR Wenk ${ }^{x, 1,4,6}$
}

Parkinson's disease (PD) is a neurodegenerative disorder marked by the selective degeneration of dopaminergic neurons in the nigrostriatal pathway. Several lines of evidence indicate that mitochondrial dysfunction contributes to its etiology. Other studies have suggested that alterations in sterol homeostasis correlate with increased risk for PD. Whether these observations are functionally related is, however, unknown. In this study, we used a toxin-induced mouse model of PD and measured levels of nine sterol intermediates. We found that lanosterol is significantly $(\sim 50 \%)$ and specifically reduced in the nigrostriatal regions of 1-methyl-4-phenyl-1,2,3,6-tetrahydropyridine-treated mice, indicative of altered lanosterol metabolism during PD pathogenesis. Remarkably, exogenous addition of lanosterol rescued dopaminergic neurons from 1-methyl-4-phenylpyridinium (MPP + )induced cell death in culture. Furthermore, we observed a marked redistribution of lanosterol synthase from the endoplasmic reticulum to mitochondria in dopaminergic neurons exposed to MPP +, suggesting that lanosterol might exert its survival effect by regulating mitochondrial function. Consistent with this model, we find that lanosterol induces mild depolarization of mitochondria and promotes autophagy. Collectively, our results highlight a novel sterol-based neuroprotective mechanism with direct relevance to PD.

Cell Death and Differentiation (2012) 19, 416-427; doi:10.1038/cdd.2011.105; published online 5 August 2011

Parkinson's disease (PD) is a movement disorder marked by the selective degeneration of dopaminergic neurons in the nigrostriatal pathway. About $5-10 \%$ of PD are genetically linked with mutations in genes such as leucine-rich repeat kinase 2 (LRRK2), alpha-synuclein, PTEN-induced putative kinase 1 (PINK1), Parkin and DJ-1. ${ }^{1}$ These genetic PD cases, while rare, have provided insights into mechanisms of $P D$ pathogenesis and many of which point toward mitochondrial dysfunction. ${ }^{1-3}$ For example, PINK1, Parkin and DJ-1 control clearance of mitochondria by mitophagy in response to cellular stress. ${ }^{3}$ Mitochondrial defects are also seen in idiopathic PD, whereby the catalytic activity of brain mitochondrial complex I is compromised. ${ }^{4}$ Finally, environmental toxins that affect complex I, such as 1-methyl-4-phenyl1,2,3,6-tetrahydropyridine (MPTP), ${ }^{5}$ can also induce Parkinsonism. The active metabolite of MPTP, 1-methyl-4phenylpyridinium $(\mathrm{MPP}+)$, selectively enters dopaminergic neurons via the dopamine transporter, inhibits mitochondrial complex I and eventually induces clinical symptoms reminiscent of PD.

As MPTP/MPP + toxicity emulate PD symptoms, it has been widely used in animal and cellular models to study neuronal cell death and to screen for neuroprotective agents. Of the neuroprotective metabolites identified, many are found in mitochondria, including L-carnitine, creatine and coenzyme $\mathrm{Q}$ (CoQ)-10. ${ }^{6}$ Although their precise protective mechanisms are still poorly understood, some evidence suggests that they act as mitochondrial uncouplers. ${ }^{7}$ Consistent with this model, uncoupling proteins (UCPs) are protective in the MPTP model of $P D,{ }^{8,9}$ and their expression is downregulated in DJ-1 knockout mice. ${ }^{10}$ Neuronal cell death induced by glutamate toxicity can also be rescued by mitochondrial uncouplers. ${ }^{11}$

More recently, misregulation of sterol metabolism has also been implicated in PD. In clinical studies, elevation of

\footnotetext{
${ }^{1}$ Department of Biological Sciences, National University of Singapore, Singapore; ${ }^{2}$ Department of Neurology, Center for Motor Neuron Biology and Disease, Columbia University, New York, USA; ${ }^{3}$ Program in Neuroscience and Neurobehavioral Disorders, DUKE-NUS Graduate Medical School, Singapore; ${ }^{4}$ Department of Biochemistry, Yong Loo Lin School of Medicine, National University of Singapore, Singapore; ${ }^{5}$ Department of Physiology, Yong Loo Lin School of Medicine, National University of Singapore (NUS), Singapore and ${ }^{6}$ Swiss Tropical and Public Health Institute, University of Basel, Socinstrasse 57, Basel, Switzerland

*Corresponding authors: L Lim or MR Wenk, Department of Biochemistry, Yong Loo Lin School of Medicine, National University of Singapore, Centre for Life Sciences, 28 Medical Drive \# 04-26D, Singapore 117456, Singapore. Tel: + 656516 3624; Fax: + 656777 3271; E-mail: g0601115@nus.edu.sg or markus_wenk@nuhs.edu.sg ${ }^{7}$ Current address: Illawarra Health and Medical Research Institute, University of Wollongong, Wollongong, New South Wales, Australia

Keywords: lanosterol; Parkinson's disease; mitochondrial membrane potential; lipids; dopaminergic neruons

Abbreviations: ATP, adenosine triphosphate; AV, autophagosome vacuole; CCCP, $m$-chlorophenylhydrazone; CCD, charge-coupled device; cdk5, cyclin-dependent kinase 5; CoQ, coenzyme Q/ Ubiquinone; DIV, days in vitro; DJ-1, (PARK7) Parkinson disease 7; ER, endoplasmic reticulum; GC-MS, gas chromatography-mass spectrometry; GSK-3 $\beta$, glycogen synthase kinase 3 beta; HPLC, high-pressure liquid chromatography; JC-1, 5,5',6,6'-tetrachloro-1,1',3,3'-tetraethylbenzimidazolocarbocyanine iodide; KDEL, ER retention sequence (lys-asp-glu-leu); LC3, microtubule-associated protein light chain 3; LDL-C, low-density lipoprotein cholesterol; LRRK2, leucine-rich repeat kinase 2; LSS, lanosterol synthase; MPP + , 1-methyl-4-phenylpyridinium; MPTP, 1-methyl-4-phenyl-1,2,3,6-tetrahydropyridine; MRM, multiple reaction monitoring; $\mathrm{p35}$, cdk5 activator protein with $35 \mathrm{kDa}$; PBS, phosphate-buffered saline; PC, phosphatidylcholine; PD, Parkinson's disease; PINK1, PTENinduced putative kinase 1; SREBP2, sterol regulatory element-binding protein 2; TH, tyrosine hydroxylase; TOMM20, translocase of outer mitochondrial membrane 20; TUJ1, neuron-specific class III beta tubulin; UCP, uncoupling protein; $\Delta \psi$, mitochondrial membrane potential

Received 01.3.11; revised 30.6.11; accepted 04.7.11; Edited by N Bazan; published online 05.8.11
} 
low-density lipoprotein cholesterol (LDL-C) in serum correlates with higher prevalence of $\mathrm{PD},{ }^{12}$ whereas higher serum levels of total cholesterol are associated with a decreased risk of PD. ${ }^{13}$ Biochemical studies showed that alpha-synuclein, the major component of Lewy bodies found in PD brains, binds to cholesterol with high affinity, ${ }^{14}$ and its aggregation is accelerated in presence of oxidized cholesterol. ${ }^{15}$ In a separate line of investigation, oxidative stress has been shown to increase levels of lanosterol, a cholesterol precursor, in mitochondria and several other intracellular compartments of macrophages, suggesting that this sterol metabolite may be part of a global cellular response to stress. ${ }^{16}$ Yet, how sterol metabolism is altered in the brains of PD patients, or which sterol metabolites, if any, have an impact on the survival of dopaminergic neurons is unknown.

Our study investigated the role of sterol metabolism in the MPTP/MPP + model of PD. Our results provide evidence for the specific role of lanosterol as a neuroprotective agent in dopaminergic neurons. We also show that lanosterol induces mild uncoupling of mitochondria and promotes autophagy, two events that have been previously linked to neuroprotection in various models of PD. To our knowledge, our work provides the first link between sterol metabolism and mitochondrial function, and identifies lanosterol as a potential therapeutic agent for PD.

\section{Results}

Lanosterol levels are decreased in the striatum and ventral midbrain of MPTP-injected mice. To determine if sterol metabolism is altered in a rodent model of PD, we measured levels of sterol metabolites in the striatum and ventral midbrain of control and MPTP-treated mice by gas chromatography-mass spectrometry (GC-MS). This acute MPTP injection regime results in about $35 \%$ loss of dopaminergic neurons after $48 \mathrm{~h} .{ }^{17}$ Of the nine metabolites analyzed, we found that lanosterol, the first cyclic sterol, was reduced by $\sim 50 \%$ in affected areas (Figure 1). Owing to the specific and highly significant reduction in the levels of lanosterol in the affected areas of MPTP-treated animals, we reasoned that lanosterol might be important for dopaminergic neuronal survival.

Lanosterol protects dopaminergic neurons from MPP + -induced cell death. We asked whether exogenous addition of lanosterol protects dopaminergic neurons against MPP + induced cell death. Primary postnatal neuron cultures from the ventral midbrain were treated with MPP +, and the survival of dopaminergic neurons was determined in the absence or presence of exogenously added lanosterol. In all, $48 \%$ of dopaminergic neurons survived on treatment with MPP + under these culture conditions (Figures $2 \mathrm{a}$ and $\mathrm{C}$ ). Co-incubation of MPP + with phosphatidylcholine (PC, vehicle control) or cholesterol did not improve survival of dopaminergic neurons (Figures $2 \mathrm{~b}$ and $\mathrm{c}$ ). In contrast, cotreatment of the cultures with MPP + and lanosterol increased survival of dopaminergic neurons to $76 \%$ (Figures $2 b$ and $c$ ). Thus, lanosterol, but not cholesterol or PC, rescues dopaminergic neurons from MPP +-induced cell death.

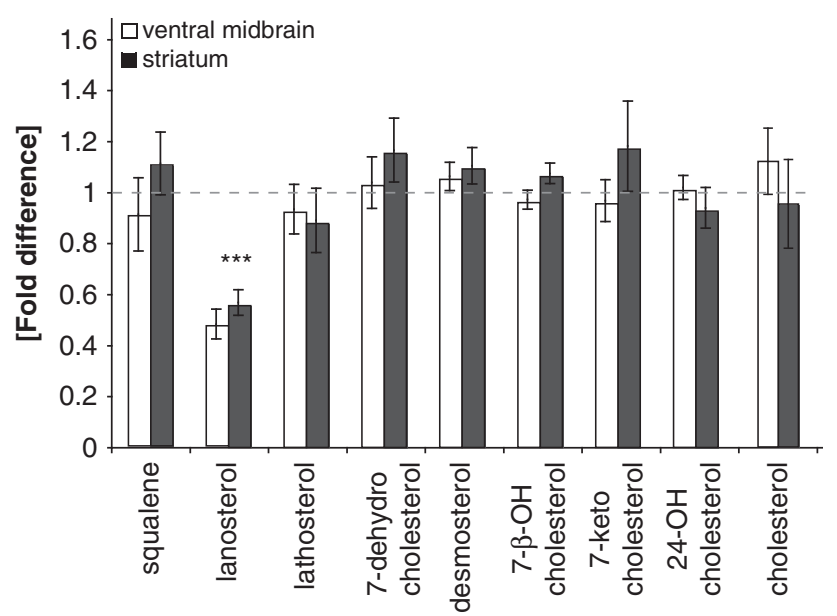

Figure 1 Lanosterol is the only sterol specifically depleted in affected brain areas of mice treated with MPTP. C576B6 mice were treated with the acute schedule of MPTP injections. At $48 \mathrm{~h}$ after the last dose of MPTP, the ventral midbrain and striatum were dissected, and lipids were extracted for analysis of sterol intermediates by GC-MS. Fold changes are plotted on the $y$ axis, which represent the average levels from MPTP-treated animals $(n=4)$ normalized to average levels from saline-treated (control) animals $(n=6)$ for each metabolite. Error bars represent S.E.M. In both brain regions, the levels of lanosterol are reduced significantly in MPTP-treated animals. ${ }^{\star \star \star} P<0.001$

To determine if addition of lanosterol in culture affects other sterol intermediates, we analyzed an array of metabolites (squalene, lanosterol, lathosterol, 7-dehydrocholesterol, desmosterol, cholesterol, 7- $\beta$ hydroxycholesterol, 7-ketocholesterol and 24-hydroxycholesterol) in extracts from ventral midbrain cultures by GC-MS. In cells treated with lanosterol, we observed a 12-fold increase in lanosterol levels relative to control (no treatment, Figures $2 d$ and $e$ ) and no detectable effect on other sterol metabolites tested (Figure 2e), suggesting that cells have a high capacity to accumulate lanosterol, consistent with previous findings in macrophages. ${ }^{16}$ This is not the case for cholesterol, as we did not detect any changes in cholesterol levels on exogenous addition of cholesterol, perhaps because neuronal cholesterol homeostasis is tightly regulated. ${ }^{18}$ In addition to the metabolites indicated above, we also tested the impact of lanosterol and cholesterol addition on levels of ubiquinones, isoprenoidderived electron carriers in mitochondria, and found no significant differences (Figures $3 a$ and $b$ ).

In these experiments, postnatal dopaminergic neurons were grown in direct contact with a glia feeder. It is therefore possible that neuroprotection is indirectly mediated by a modification of astrocyte physiology. To address whether lanosterol addition leads to a specific increase in lanosterol concentration in neurons, we repeated these experiments using hippocampal neurons cultured according to Banker's method, ${ }^{19}$ which allows physical separation of neurons and astrocytes. We found that lanosterol levels increased in both neurons and astrocytes to a similar extent on treatment with exogenous lanosterol (data not shown). Although we cannot rule out the possible contribution of astrocytes in lanosterolmediated neuroprotection, our results indicate that lanosterol levels are significantly elevated in neurons, arguing for a direct role of lanosterol in promoting survival of dopaminergic neurons. 
a

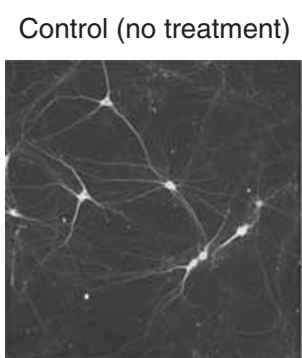

$10 \mu \mathrm{M} \mathrm{MPP}+$

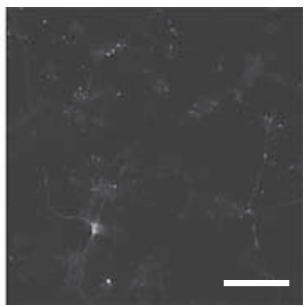

b $\quad 10 \mu \mathrm{MMPP}+\&$ $5 \mu \mathrm{M}$ PC

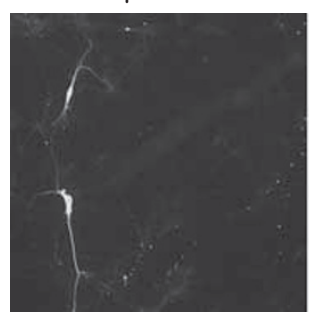

$10 \mu \mathrm{M} M P P+\&$ $5 \mu \mathrm{M}$ Lanosterol

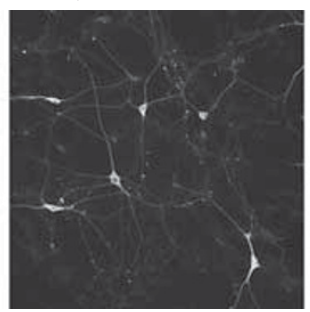

d

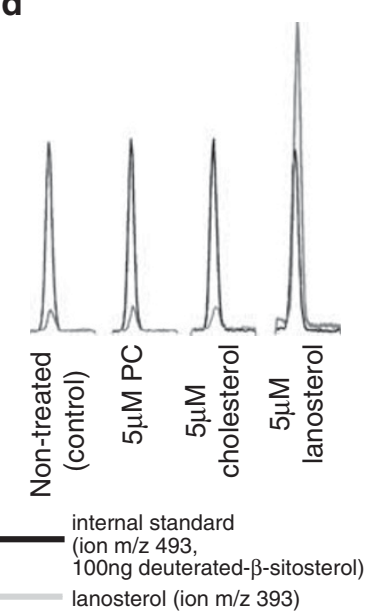

e

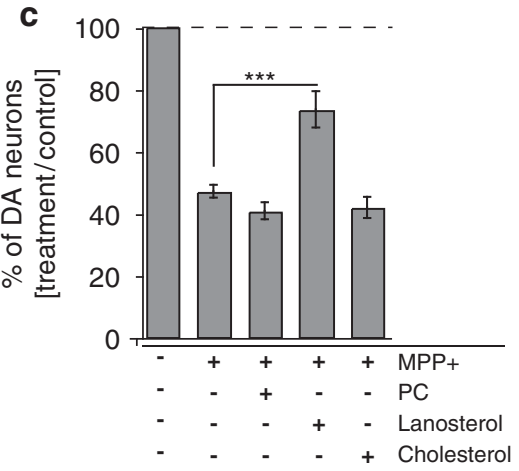

10uM MPP+ \&

$5 \mu \mathrm{M}$ Cholesterol
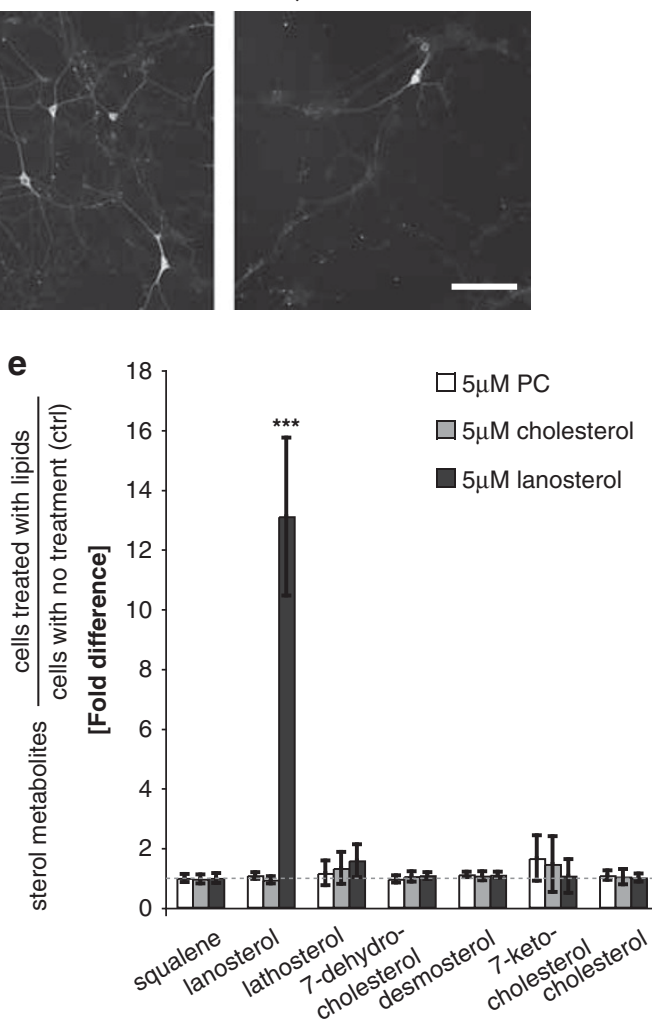

Figure 2 Lanosterol rescues dopaminergic neurons in MPP +-treated ventral midbrain cultures. Fluorescence images of primary ventral midbrain cultures (DIV7) stained with anti-TH, a marker for dopaminergic neurons. (a) Control cells (no treatment) and cells treated with $10 \mu \mathrm{M} \mathrm{MPP}+$; (b) co-treated cells for $24 \mathrm{~h}$ with $10 \mu \mathrm{M}$ MPP + and $5 \mu \mathrm{M}$ of phosphatidylcholine (PC, left panel), lanosterol (middle panel) or cholesterol (right panel). Scale bars in (a) and (b) represent $200 \mu \mathrm{m}$. (c) Plot of fold changes of each treatment relative to control. $y$ axis shows the average percentage of $\mathrm{TH}+$ neurons from each treatment condition divided by the average percentage of $\mathrm{TH}+$ neurons in control. Error bars represent S.E.M. from four to five independent experiments. ${ }^{\star * \star} P<0.001$ between lanosterol/MPP + and MPP + . (d) GC-MS profiles of lanosterol $(\mathrm{m} / \mathrm{z} 393$, light grey) and the internal standard (deuterated $\beta$-sitosterol, $\mathrm{m} / \mathrm{z} 493$, black) in ventral midbrain cultures incubated for $24 \mathrm{~h}$ with various lipid treatments. (e) Cellular levels of sterol intermediates following various treatments normalized to control (no treatment). Fold changes plotted in y axis represent the levels of each metabolite in each treatment condition normalized to control. Error bars represent S.E.M. from three independent experiments. ${ }^{* \star} P<0.001$ between lanosterol treatment and control

To explore the mechanisms underlying lanosterol-mediated neuroprotection, we next investigated the effects of MPP + and lanosterol on various signaling pathways previously implicated in cellular metabolism and neurodegeneration. First, we examined the expression level of sterol response element-binding protein (SREBP2, Figure 4a), a transcription factor reported to exacerbate neuronal degeneration. ${ }^{20}$ We found that MPP + induced a modest increase in
SREBP2 levels, which was not rescued by lanosterol addition (Figure $4 \mathrm{a}$, top panel). We also checked for levels of cdk5 activator protein with $35 \mathrm{kDa}$ (p35), the activator of cyclin-dependent kinase 5 (cdk5), because the genetic ablation of p35/cdk5 confers neuroprotection in the MPTP model. ${ }^{21}$ Although we observed a decrease in p35 levels in MPP +-treated cells, addition of lanosterol did not restore p35 expression to control levels (Figure $4 a$, second panel 
a

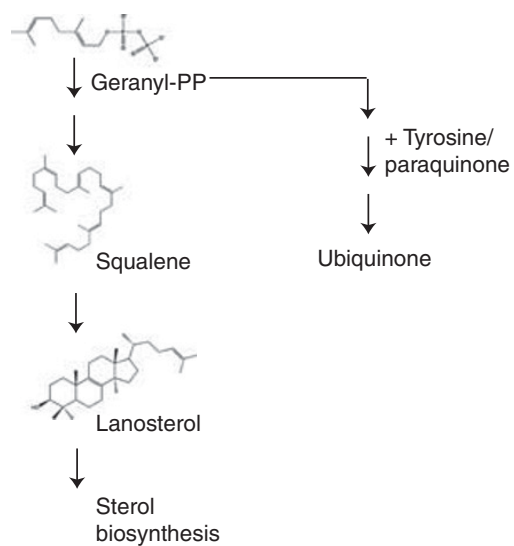

b

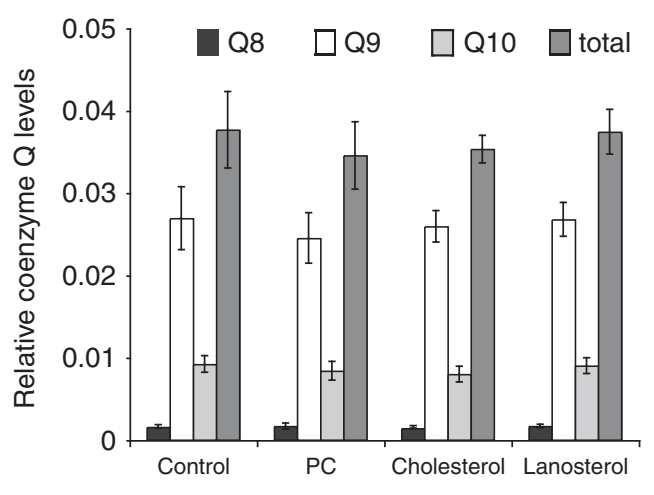

Figure 3 Measurements of ubiquinone on addition of lipids. (a) Simplified pathway to show the cross-talk between sterol and ubiquinone biosynthesis in mammalian cells. Both pathways use isoprenyl units as precursors. (b) Plot of ubiquinone/CoQ levels normalized to total cholesterol levels in neuronal cultures from various treatment conditions. Error bars represent S.E.M. over four independent experiments

from top). Consistent with previous findings implicating glycogen synthase kinase 3 beta (Gsk-3 $\beta$ ) (Ser9) phosphorylation in PD pathogenesis, ${ }^{22}$ we found increased phosphoGsk-3 $\beta$ in MPP + -treated cells. However, this increase was not affected by the addition of lanosterol (Figure $4 \mathrm{a}$, third panel from top).

Finally, we examined the levels of lanosterol synthase (LSS), the enzyme that catalyzes cyclization of oxidosqualene, the rate limiting step in lanosterol synthesis. ${ }^{23}$ The levels of LSS did not markedly change on MPP + addition (Figure 4a, fourth panel from top). Interestingly, however, we observed a different localization pattern of LSS in MPP + -treated dopaminergic neurons. LSS immunostaining appeared much more punctate after MPP + treatment (Figure 4b), suggesting drug-induced redistribution of LSS to a different intracellular compartment. We thus proceeded to examine the subcellular localization of LSS in control and $\mathrm{MPP}+$-treated dopaminergic neurons.

Redistribution of LSS in dopaminergic neurons on MPP + insult. LSS is a membrane-associated enzyme, which is targeted to the cytoplasmic leaflet of the endoplasmic reticulum $(E R) .{ }^{24}$ Biochemical analysis of purified fractions from mouse liver showed that the bulk of LSS is indeed associated with the microsomal/ER fractions and that minor amounts are found in purified mitochondria (Figure 4c).

Consistent with our biochemical fractionation data, we found that LSS colocalized with an ER marker (ER retention sequence (lys-asp-glu-leu) (KDEL)) in both dopaminergic and non-dopaminergic neurons (Figure 5a). Extensive overlap between LSS and the ER marker was observed for both dopaminergic and non-dopaminergic neurons $\left(R^{2}=0.83 \pm\right.$ 0.01 and $0.84 \pm 0.01$, respectively). Less overlap was found between LSS and translocase of outer mitochondrial membrane 20 (TOMM20) (a mitochondrial marker) for both dopaminergic and non-dopaminergic neurons $\left(R^{2}=0.70 \pm\right.$ 0.02 and $0.69 \pm 0.02$, respectively, Figure $5 a$, second panel from the top). This pattern changed noticeably in dopaminergic neurons treated with MPP + . The colocalization of LSS with TOMM20 was markedly increased $\left(R^{2}=0.856 \pm 0.01\right.$,
Figure $5 \mathrm{a}$, bottom last panel), with concomitant reduction in the overlap of LSS with $\operatorname{KDEL}\left(R^{2}=0.69 \pm 0.02\right.$, Figure $5 \mathrm{a}$, second panel from the bottom). Translocation of LSS from the ER to mitochondria was also observed in dopaminergic neurons co-treated with MPP + and lanosterol (Figure 5b). MPP + did not, however, affect LSS localization in nondopaminergic neurons (Figure 5b), consistent with the inability of these cells to take up MPP + . These data indicate that a significant fraction of LSS redistributes from the ER to mitochondria in dopaminergic neurons exposed to MPP +, suggesting that LSS (and its product lanosterol) may have a role in regulating mitochondrial function.

Lanosterol uncouples mitochondria in dopaminergic neurons. An important aspect of mitochondrial physiology can be assessed by measuring the mitochondrial membrane potential $(\Delta \psi)$, which is the electrical and chemical gradient that drives protons across the inner membrane during electron transport and oxidative phosphorylation. This process is never completely 'coupled', allowing a fraction of electrons and protons to be transported without concurrent production of adenosine triphosphate (ATP). We first examined the impact of lanosterol on $\Delta \psi$ in hippocampal neurons using the ratiometric voltage-sensitive fluorescent dye, 5,5',6,6' 'tetrachloro-1,1',3,3'-tetraethylbenzimidazolocarbocyanine iodide (JC-1). Using time-lapse, multi-positioning imaging to capture about 3-4 fields in 30-s intervals, we measured changes of $\Delta \psi$ on lipids addition by the ratiometric analysis of JC-1 red to green emission. As expected, treatment of neurons with $200 \mathrm{nM} m$-chlorophenylhydrazone (CCCP), a known uncoupler of oxidative phosphorylation, induced an immediate and sharp reduction of the mitochondrial membrane potential $(\sim 25 \%$ within $30 \mathrm{~s}$; Figure 6a). Under these same experimental conditions, exogenous addition of lanosterol reduced the membrane potential by $\sim 20 \%$ over $15 \mathrm{~min}$, whereas PC and cholesterol had no significant effect (Figure 6b).

We repeated these experiments in ventral midbrain cultures (Figures $6 \mathrm{c}$ and $\mathrm{d}$ ). As these cultures were grown at a lower density than the hippocampal cultures, we needed to capture 10-15 fields in time-lapse multi-positioning imaging at lower 
magnification to gain enough statistical power. As a consequence, this limited time intervals to $2 \mathrm{~min}$. We observed a $\sim 20 \%$ of reduction in membrane potential on lanosterol addition (Figures $6 \mathrm{c}$ and $\mathrm{d}$ ), consistent with the results obtained in hippocampal neurons.
As MPP + uptake depends on ATP levels, ${ }^{25}$ it is possible that the addition of lanosterol causes mitochondrial depolarization, and subsequently ATP depletion. This would, in turn, inhibit MPP + uptake in the mitochondria. To determine if lanosterol alters ATP levels, we measured levels of ATP in a

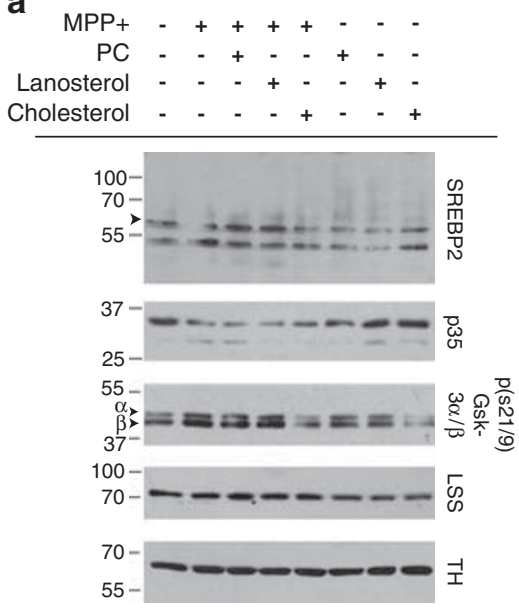

b

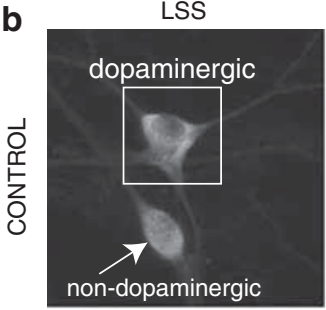

LSS

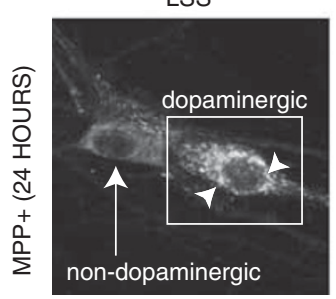

$\mathrm{TH}$

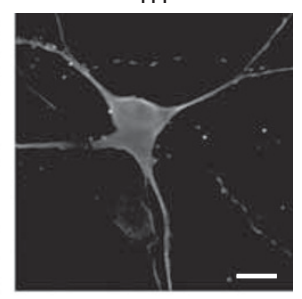

$\mathrm{TH}$

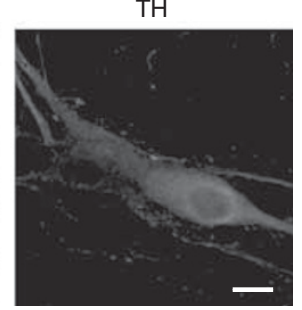

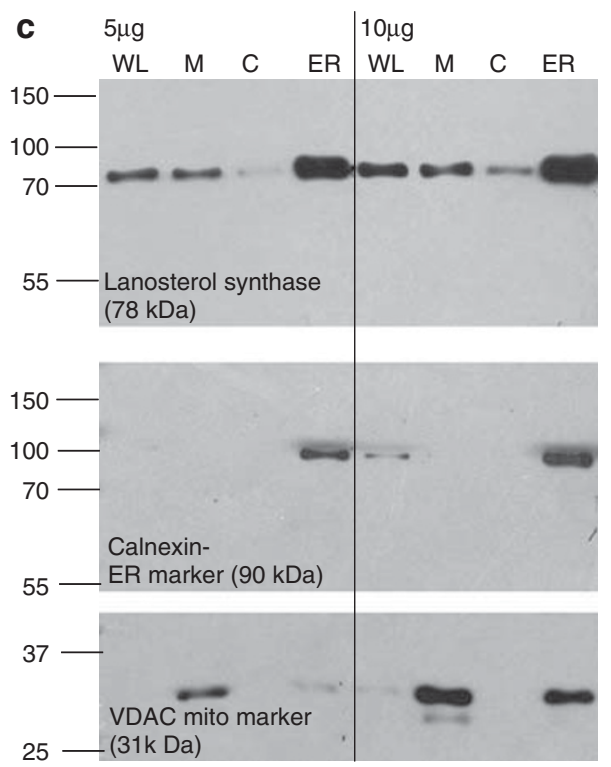

Figure 4 Analyses of SREBP2, Gsk-3 $\beta$, p35/cdk5 and LSS in ventral midbrain treated with lipids and MPP + . (a) Immunoblot analyses of several factors previously linked to neuroprotection in PD and/or MPTP treatment. (b) Immunofluorescence images of ventral midbrain neurons stained with LSS and TH treated with or without MPP + . LSS appears more punctate in TH + neurons on MPP + treatment. (c) Subcellular fractionation of liver tissue. M, mitochondrial, ER, microsome/ER, C, cytosolic, WL, fractions were purified from whole liver. Immunoblot of calnexin (ER maker) and VDAC/porin (mitochondrial marker) were used to assess the purity of each fraction. LSS showed a clean single band at the expected weight of $78 \mathrm{kDa}$ and is enriched in the ER fraction. A small amount of LSS is also detected in the mitochondrial fraction

Figure 5 LSS is redistributed from ER to mitochondria in dopaminergic neurons on addition of MPP + . (a) Confocal images of ventral midbrain neurons stained with TH (white), LSS (green), either KDEL (red, first and third panels from the top) or TOMM20 (red, second and fourth panels from the top) of ventral midbrain neurons in control and MPP + treatment. White boxes show dopaminergic neurons as determined by TH + staining. Right panels show pixel intensity correlation plots of LSS with either KDEL or TOMM20 in dopaminergic neurons. Scale bar represents $10 \mu \mathrm{m}$. (b) Average $R^{2}$ values (a measure of colocalization) of two classes of neurons in control and MPP + -treated conditions. In control, $R^{2}$ values from co-staining of KDEL-LSS, $n=26$ and $n=27$, TOMM20-LSS, $n=20$ and $n=21$, were assessed for dopaminergic and nondopaminergic neurons respectively. In MPP + -treated cells, $R^{2}$ values from co-staining of KDEL-LSS, $n=20$ and $n=20$, for TOMM20-LSS, $n=18$ and $n=17$, were assessed for dopaminergic and non-dopaminergic neurons respectively. In MPP + /lanosterol co-treated cells, $R^{2}$ values from co-staining of KDEL-LSS, $n=18$ and $n=23$, for TOMM20-LSS, $n=19$ and $n=18$, were assessed for dopaminergic and non-dopaminergic neurons respectively. ${ }^{* \star \star \star} P<0.00001$ 
neurons treated with PC, cholesterol or lanosterol. We did not see any significant changes in the levels of ATP across all treatment conditions (Supplementary Figure S1), indicating that lanosterol is unlikely to inhibit MPP + uptake. Thus, one mechanism by which lanosterol could mediate neuroprotection is through the uncoupling of mitochondria.
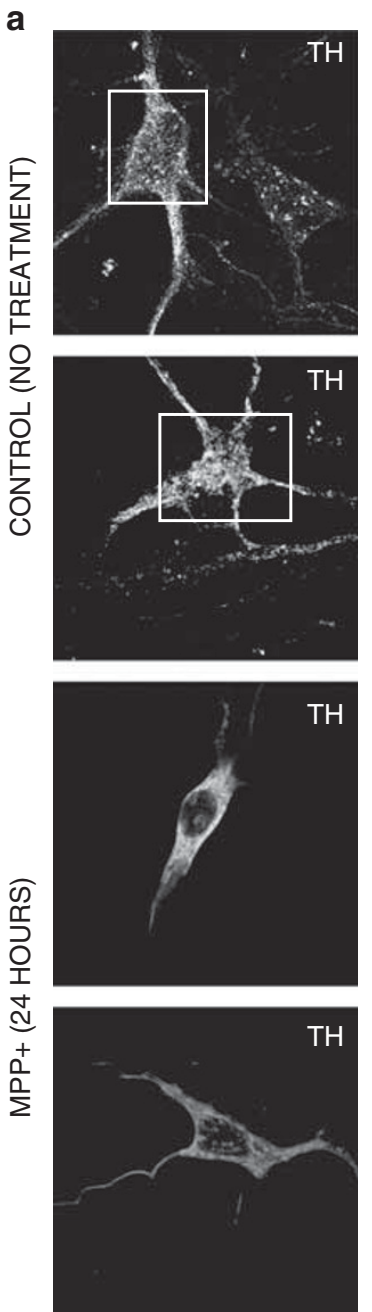

$\mathrm{TH}$
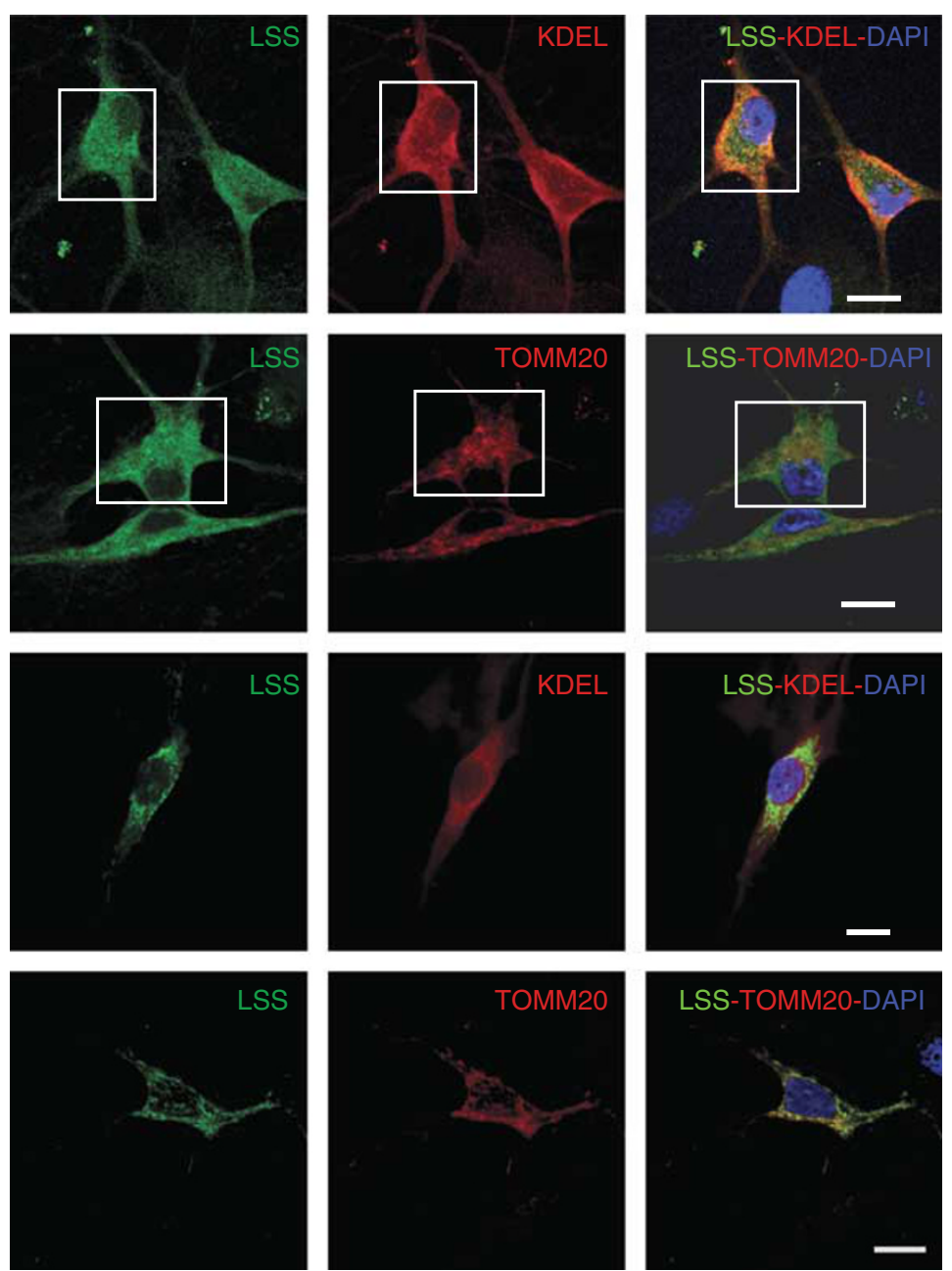

b $1.00 \square$ TOMM20-LSS KDEL-LSS

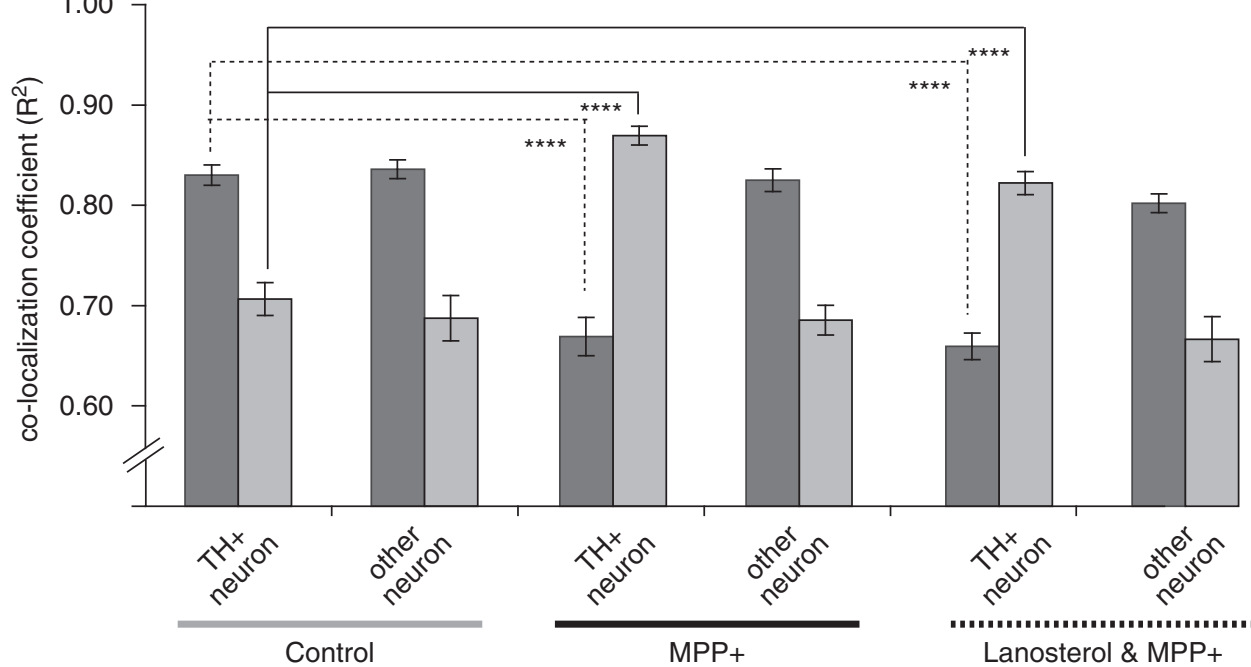

Control

Lanosterol \& MPP+ dopaminergic neuron
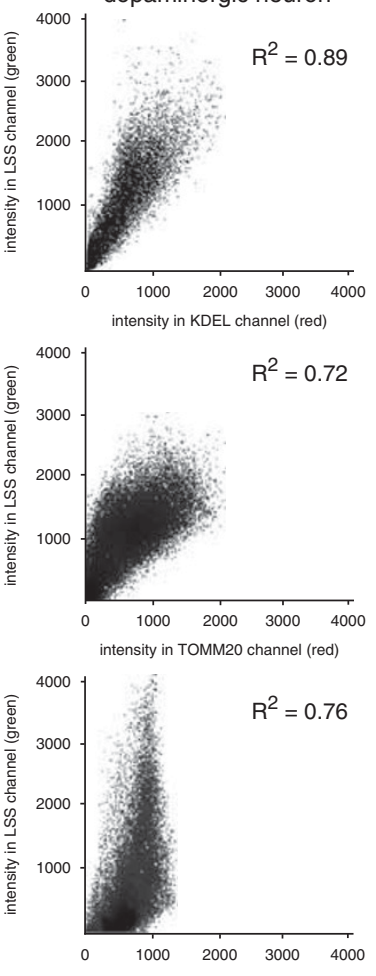

intensity in KDEL channel (red)

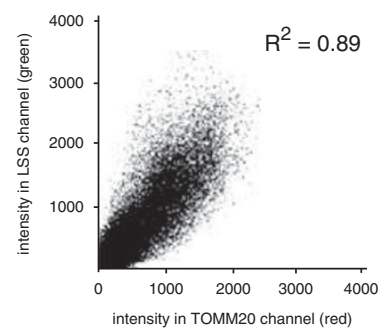



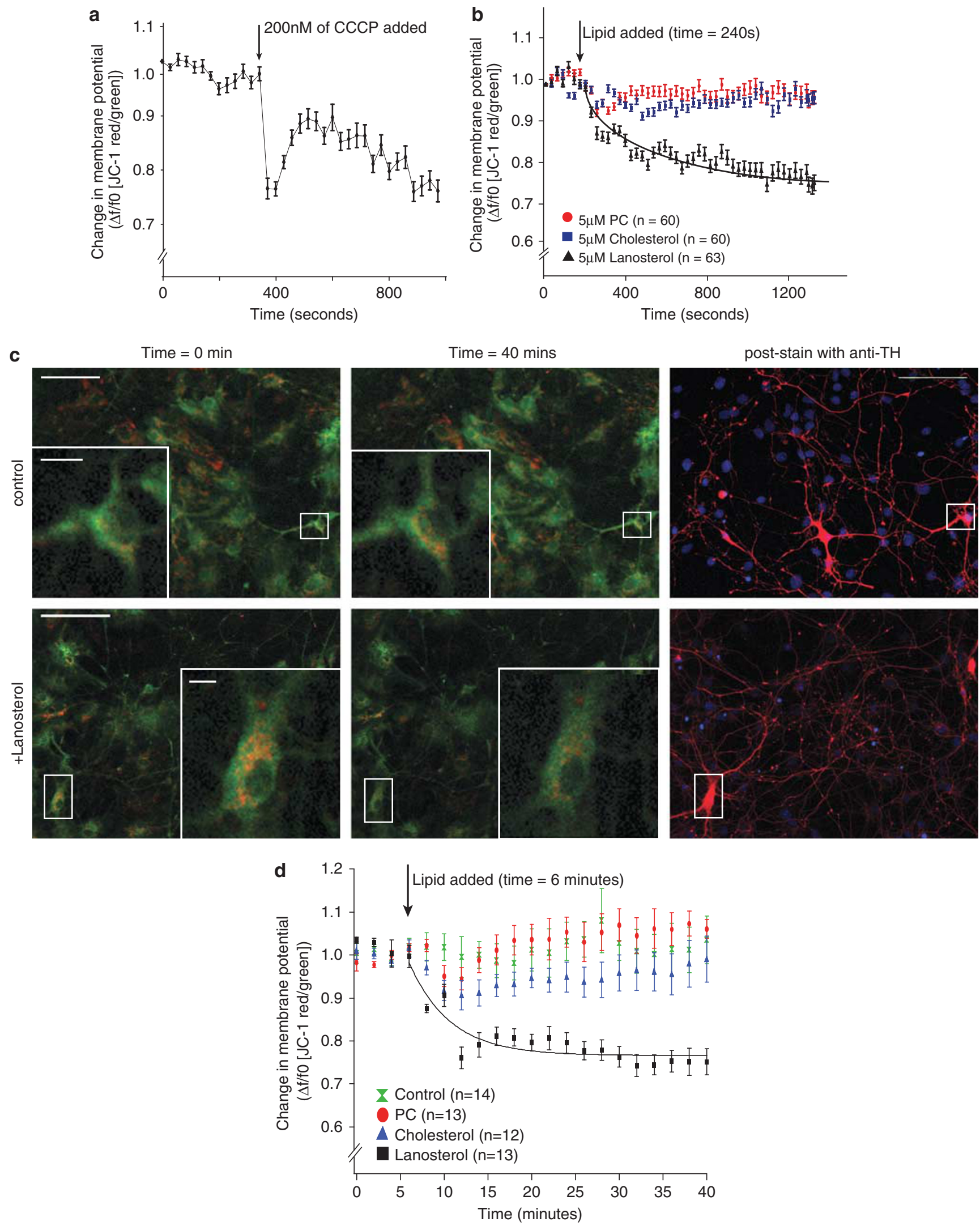
Lanosterol increases autophagy in dopaminergic neurons. Previous studies have shown that the loss of mitochondrial membrane potential can initiate the autophagic degradation of damaged mitochondria. ${ }^{26,27}$ Gene linked to familial forms of PD, such as PINK1 and Parkin, are thought to regulate this process, and PD-associated Parkin mutations cause a decrease in mitophagy in mammalian cell lines. ${ }^{28}$ We thus asked if lanosterol mediates autophagosome formation in dopaminergic neurons. Using microtubuleassociated protein light chain 3 (LC3) as a marker for autophagy, we quantified both the size and number of autophagic vacuoles (AVs) in primary ventral midbrain neurons on MPP +/lanosterol treatment. Similar to other studies, $^{29}$ we found that addition of MPP + in primary dopaminergic neurons increased the number of AVs by about 2.5-fold (Figure 7). There is also about a $75 \%$ increase in the average size of AVs with MPP + treatment. Remarkably, when neurons were exposed to lanosterol alone, we observed a similar increase in AV size and number. Co-treatment of MPP + and lanosterol led to an additive effect on autophagy, with significant increases in both the number and size of AVs, compared with MPP + or lanosterol alone (Figure 7).

Next, we addressed whether lanosterol has a specific effect on axonal mitophagy. In PD, axons of dopaminergic neurons progressively degenerate and 'die back', in a process that may be accelerated by mitochondrial dysfunction and involve mitophagy. For this, we grew hippocampal neurons in microfluidic chambers to segregate axons from neuronal cell bodies and dendrites. ${ }^{30}$ Mitochondria were visualized by expression of the fluorescent reporter MitoRed and neurons were immunostained for endogenous LC3. We found a small but significant increase in colocalization of MitoRed with LC3 (Figure 8) on lanosterol treatment, suggesting an increase in axonal mitophagy. Taken together, these results suggest that the protective effects of lanosterol are mediated by mitochondria uncoupling, and subsequent clearance of damaged mitochondria.

\section{Discussion}

The etiology of PD implicates several factors, including mitochondrial dysfunction and misregulation of sterol metabolism. Evidence implicating impaired mitochondrial function in PD is substantial. This evidence is based on (1) the identification of rare PD-associated mutations in genes that affect mitochondrial function such as the putative kinase, PINK1 (PARK6), the E3 ligase Parkin (PARK2) and DJ-1 (PARK7); (2) similarities between PD and clinical symptoms that arise on exposure to the neurotoxin MPTP, a complex I inhibitor; and (3) a significant decrease in complex I/II activity in the platelets of patients with PD. ${ }^{31}$
On the other hand, evidence for a role of aberrant sterol metabolism in PD is rather controversial. For example, lower levels of serum LDL-C are considered a risk factor for $P D,{ }^{12,32}$ but it is unclear how serum LDL-C relates to levels of cholesterol in the brain. Statins, which lower serum cholesterol levels by inhibiting 3-hydroxy-3-methyl-glutaryl-coenzyme $A$ reductase, have been used to treat $P D$, but there are many unresolved questions regarding their benefits (see review in Becker and Meier $^{33}$ ).

In this study, we measured a selection of sterol metabolites in the ventral midbrain and striatum of MPTP-treated mice, two brain regions characterized by cell death and/or axonal loss in both the MPTP model as well as in PD. Our results show a highly selective reduction of lanosterol levels in these affected areas (Figure 1) and point to an alteration of lanosterol metabolism in MPTP-treated mice. We cannot rule out the possibility that lanosterol is oxidized or metabolized to di-hydrolanosterol or other products in MPTP-treated animals because we are unable to measure these oxidized metabolites.

Consistent with a role of lanosterol in PD pathogenesis, we observed an improved survival of MPP + -treated dopaminergic neurons on exogenous addition of lanosterol (Figure 2). We also demonstrated that LSS relocalized from the ER to mitochondria in dopaminergic neurons on MPP + treatment (Figures $4 b$ and 5), suggesting an increase in lanosterol synthesis in mitochondria. Interestingly, recent lipidomic analysis of macrophages during stimulation with Lipid A (a condition that leads to oxidative bursts) showed a pronounced increase in lanosterol levels in several intracellular compartments, including mitochondria, ${ }^{16}$ implicating that modulation of lanosterol metabolism may be part of a global cellular response to stress. If upregulation of lanosterol synthesis is part of a cellular defense mechanism, it is not clear why lanosterol levels drop in response to MPTP treatment. In this regard, it is interesting to note that two recent papers reported lowered lanosterol levels in the serum of patients with Alzheimer's disease, ${ }^{34}$ and in fibroblasts challenged by virus infection. ${ }^{35}$ One possible explanation for these seemingly contradicting results is that distinct types of stress differentially impact lanosterol metabolism. Perhaps, in some cases, the substantial decrease in lanosterol levels cannot be compensated by upregulation of lanosterol synthesis as part of the cell's protective response. Alternatively, we cannot exclude the possibility that translocation of LSS to mitochondria in response to MPP + is an epiphenomenon, which is not indicative of a cellular response to stress.

Our results, however, show that exogenous addition of lanosterol leads to mild uncoupling of mitochondria in dopaminergic and glutamatergic neurons, with no detectable impact on ATP levels (Figure 6 and Supplementary Figure

Figure 6 Lanosterol induces uncoupling of the mitochondria in dopaminergic neurons. Live imaging of JC-1 loaded neuronal cultures. (a) Control plot showing changes in $\mathrm{JC}-1$ red to green ratio, $\Delta \mathrm{f} / \mathrm{f}_{0}$, versus time in hippocampal neurons treated with $\mathrm{CCCP}$, a known uncoupler, $n=20$. (b) Plot of $\Delta \mathrm{f} / \mathrm{f}_{0}$ versus time in hippocampal neurons treated with different lipids, $n>60$ for each condition from three independent experiments. (c) Images of control and lanosterol-treated ventral midbrain cultures at the start of the experiment (time $=0 \mathrm{~min}$, left panels) and the end of the experiment (time $=40 \mathrm{~min}$, middle panels). Right panels represent posteriori staining of TH for identification of dopaminergic neurons. White boxes represent magnified TH + neurons. Scale bar represents $100 \mu \mathrm{m}$ in main figure and $10 \mu \mathrm{m}$ in magnified box. (d) Plot of $\Delta \mathrm{f} / \mathrm{f}_{0}$ versus time for dopaminergic neurons, $n>10$ for each condition from three independent experiments. In both types of cultures, lanosterol induces about $20 \%$ decrease in mitochondrial membrane potential 
a
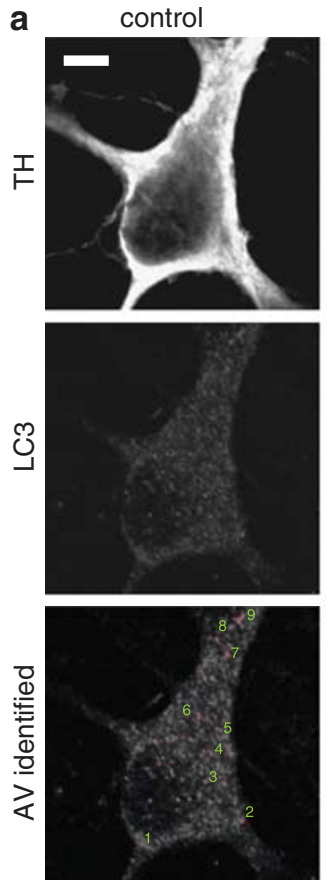

lanosterol
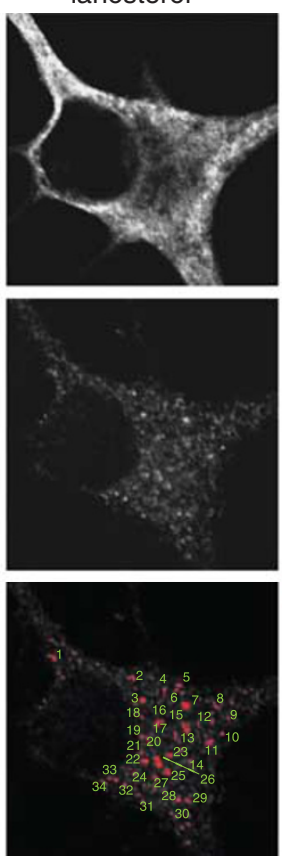

$\mathrm{MPP}+$
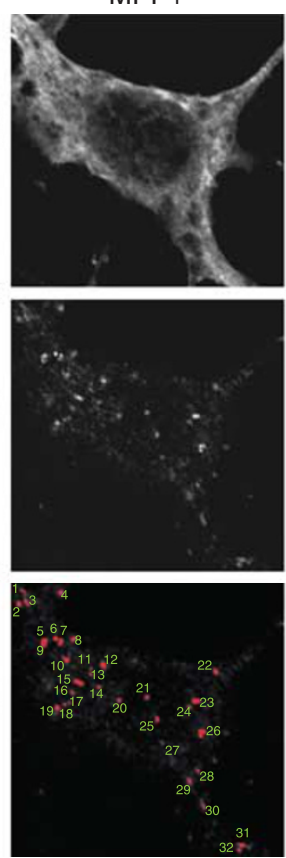

Lanosterol \& MPP+
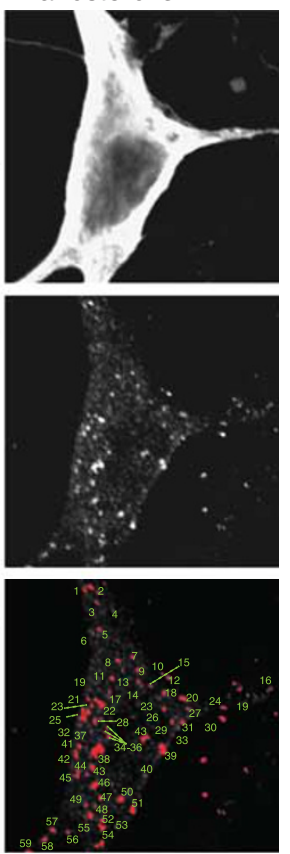

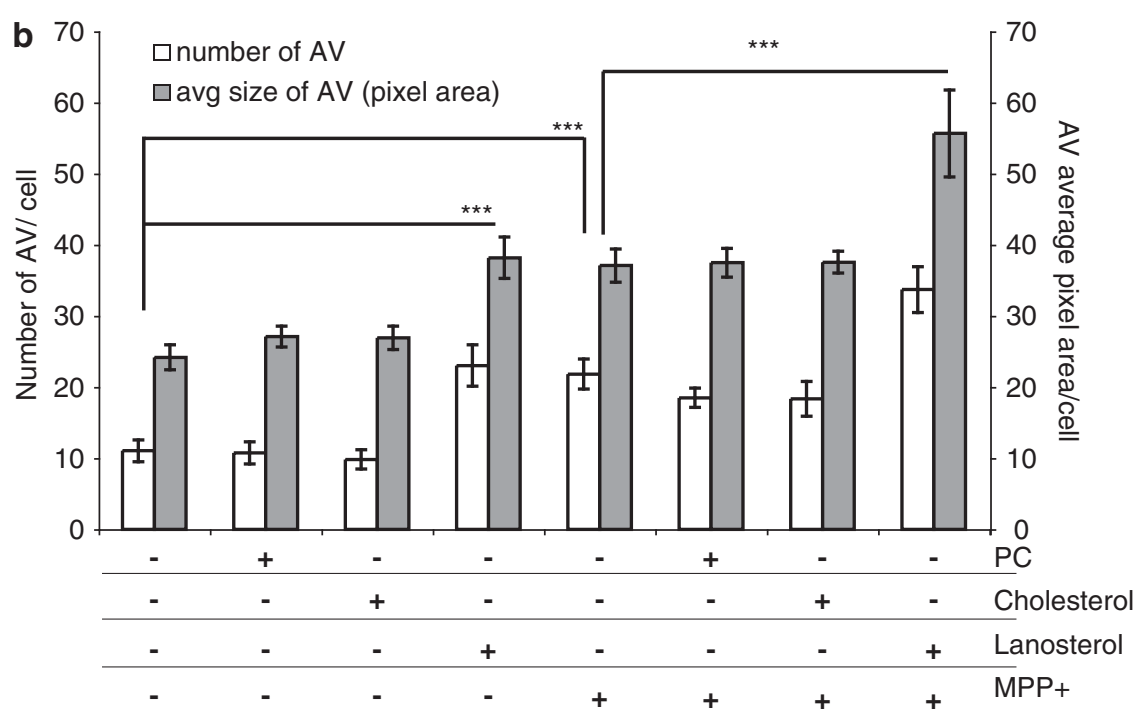

Figure 7 Lanosterol and MPP + increase the number of AVs in dopaminergic neurons. (a) Confocal images of ventral midbrain neurons stained with TH (top panels), LC3 (middle panels) and AV quantification (ImageJ software output, bottom panels) with various treatment conditions indicated on the top. Scale bar represents $10 \mu \mathrm{m}$. (b) Graphical plot showing the average number of AV identified per TH + cells and the average size of AV in pixel area during treatment with various lipids and MPP + co-treatment. For each condition, $n>40 \mathrm{TH}+$ cells from three independent experiments. Error bars represent S.E.M. ${ }^{\star \star \star} P<0.001$

S1). The mitochondria uncoupling and protective effects of lanosterol are strikingly similar to those observed with low dose of the uncoupler, FCCP, which improves cellular survival in ischemic preconditioning but has no significant impact on ATP levels. ${ }^{36}$ In the context of PD, the mitochondrial uncoupling effect of lanosterol has important implications. For example, Parkin is recruited to mitochondria via PINK1 on membrane depolarization, ${ }^{26}$ and regulates the clearance of damaged mitochondria by mitophagy in mammalian cell lines. ${ }^{37}$ In addition, the translocation of Parkin to mitochondria has etiological significance, as a number of disease- associated Parkin mutant proteins fail to translocate. ${ }^{27,38}$ Together, these data point to a role of mitochondrial uncoupling and autophagy in PD pathogenesis. In line with this model, our results reveal that lanosterol induces mitochondrial uncoupling (Figure 6) and promotes autophagy (Figures 7 and 8).

Although mitochondrial uncoupling has been shown to be neuroprotective in various models, including MPTP-induced neurodegeneration, ${ }^{7-9}$ the mechanisms involved are still unclear. Some studies have shown that uncoupling reduces superoxide species, offering an explanation for improved 
a
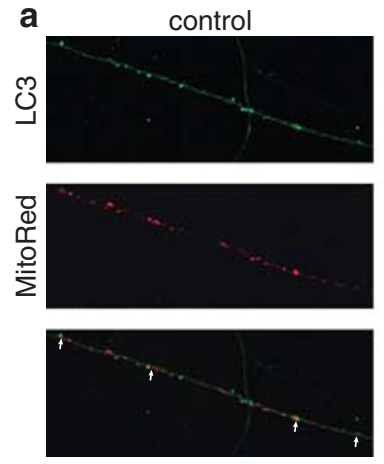

lanosterol

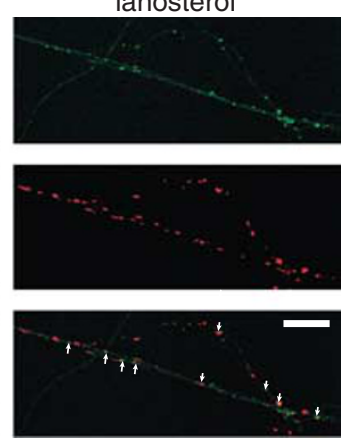

b

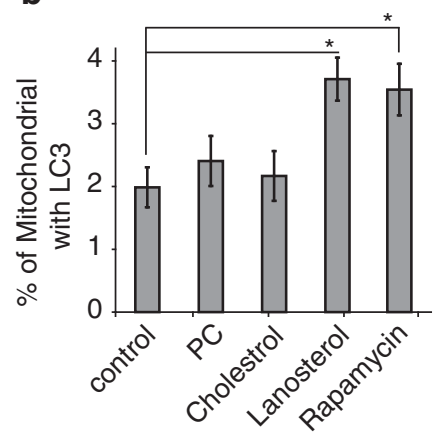

Figure 8 Lanosterol increases mitophagy in axons. (a) Confocal images of hippocamal axons stained with LC3 (top panels, green) and electroporated with MitoRed (middle panels, red). Bottom panels show of LC3 and MitoRed. White arrows represent-colocalization of the two channels. Scale bar represents $20 \mu \mathrm{m}$. (b) Graphical plot of average percentage of mitochondria with LC3-positive stains. Error bars represent S.E.M. For each condition, $n>40$ axons were assessed from three independent experiments. ${ }^{*} P<0.05$

neuronal survival in the MPTP model, because oxidative stress is thought to be the primary cause of cell death. ${ }^{8,9}$ In other studies, transient mitochondrial uncoupling is neuroprotective in glutamate-induced neurotoxicity, as it prevents uptake of calcium from the cytosol to mitochondria. ${ }^{11}$ Finally, a recent study showed that $D J-1$, a gene involved in early onset PD, regulates the expression of two UCPs (UCP4 and UCP5) and controls oxidative stress in mitochondria of dopaminergic neurons in the substantia nigra. ${ }^{10}$ Although these studies cited above have identified different modulators by which a cell/neuron alters mitochondrial membrane potential, they are in good agreement with our findings, whereby uncoupling mechanism proves to be a central regulator of cellular response to stress.

In conclusion, we report that in addition to its role as a precursor for cholesterol biosynthesis, lanosterol acts as a survival factor for dopaminergic neurons. Furthermore, our findings point to an unexpected role of this sterol metabolite in regulating mitochondrial function and autophagy, and bring sterol metabolism to the forefront of neurobiological disease.

\section{Materials and Methods}

PD animal model: MPTP injections. All procedures performed in rodents were in accordance with IACUC guidelines. MPTP injections were performed according to previously published methods, following the acute schedule. ${ }^{17}$ Briefly, C57B6 mice were given four i.p. doses of either $18 \mathrm{mg} / \mathrm{kg}$ of MPTP (Sigma-Aldrich, St. Louis, MO, USA) or saline (control) every $2 \mathrm{~h}$. Mice were decapitated $48 \mathrm{~h}$ after the last dose, and the ventral midbrain and striatum were dissected and snap-frozen for subsequent lipid extraction and GC-MS analysis. Previously published data using the same protocol showed that at this timepoint, about $35 \%$ of dopaminergic neurons have degenerated. ${ }^{17}$

Lipid standards and liposomes. Lanosterol, cholesterol, 1,2-dimyristoylsn-glycero-3-phosphocholine (DMPC or PC) and desmosterol- $d_{6}$ (all of highest purity, $>99 \%$ ) were purchased from Avanti Polar Lipids (Alabaster, AL, USA). Oxysterol standards $\alpha$-cholestane, $7 \alpha$-hydroxycholesterol, $7 \beta$-hydroxycholesterol, 7-dehydrocholesterol, 25-hydroxycholesterol and 7-ketocholesterol were obtained from Sigma (St. Louis, MO, USA). $7 \alpha$-Hydroxycholesterol- $\alpha_{7}$, $7 \beta$-hydroxycholesterol- $d_{7}, \quad \beta$-sitosterol- $\alpha_{7}, \quad$ campesterol- $\alpha_{3}$, lathosterol- $\alpha_{4}$ and 7-ketocholesterol- $d_{7}$ were purchased from CDN Isotopes (Quebec, Canada). 27-Hydoxycholesterol- $d_{5}$, 24-hydroxycholesterol and 24-hydroxycholesterol- $d_{7}$ were purchased from Medical Isotopes (Pelham, AL, USA). Deuterated standards obtained were of $>95 \%$ purity.
Lanosterol, cholesterol and PC were dissolved in chloroform/methanol (1:1). Cholesterol or lanosterol was mixed in equimolar proportion with $\mathrm{PC}$ and dried by vacuum in a Speedvac (Thermosavant, Waltham, MA, USA). The lanosterol/PC or cholesterol/PC mixture was resuspended in culture medium on the day of treatment to make a $0.5 \mathrm{mM}$ stock liposome. Each type of stock liposome was used in the neuronal cultures within a day of preparation.

Lipid extraction. For tissues and cells, extraction of lipids was performed using Bligh and Dyer method. ${ }^{39}$ Briefly, cells were washed 3-5 times with phosphatebuffered saline (PBS), scraped in $400 \mu$ lice-cold methanol and transferred to a 1.5-ml Eppendorf tube. Chloroform $(200 \mu \mathrm{l})$ was added and samples were vortex for $1 \mathrm{~min}$. Next, $300 \mu \mathrm{l}$ of $1 \mathrm{M} \mathrm{KCL}$ was added, and the homogenates were microcentrifuged at 14000 r.p.m. for $5 \mathrm{~min}$ at $4^{\circ} \mathrm{C}$ to separate phases. The lower organic phase was carefully transferred to a new Eppendorf tube. The aqueous phase was re-extracted twice with $300 \mu \mathrm{l}$ chloroform. All organic phases were pooled and dried under vacuum using a Speedvac and stored at $-80^{\circ} \mathrm{C}$ until derivatization and subsequent GC-MS analysis.

Ventral midbrain $(\sim 20-25 \mathrm{mg})$ and striatum $(\sim 15-20 \mathrm{mg})$ were dissected and tissues were homogenized using a Dounce homogenizer in $600 \mu \mathrm{l}$ ice-cold chloroform/methanol (1:2). Another $300 \mu \mathrm{l}$ chloroform was added to the homogenate followed by the addition of $450 \mu \mathrm{l}$ of $1 \mathrm{M} \mathrm{KCL}$. The homogenates were microcentrifuged at 14000 r.p.m. for $5 \mathrm{~min}$ at $4{ }^{\circ} \mathrm{C}$. The lower organic phase was carefully transferred to a new Eppendorf tube, and the aqueous phase was reextracted twice with $300 \mu \mathrm{l}$ chloroform. All organic phases were pooled and dried under vacuum in a Speedvac. Dried samples were then stored at $-80^{\circ} \mathrm{C}$ until derivatization and subsequent analyses.

Sample preparation for GC-MS. Briefly, dried lipid extracts were resuspended in chloroform/methanol $(1: 1)$ to a concentration of $0.1 \mathrm{mg}$ tissue $/ \mu \mathrm{l}$ solvent. A $20-\mu$ l sample of lipid extract was removed and completely dried in a glass vial. For each sample, we added a mixture of heavy isotopes: $40 \mathrm{ng}$ of $7 \alpha$-hydroxycholesterol- $\alpha_{7}, 40 \mathrm{ng}$ of $7 \beta$-hydroxycholesterol- $\alpha_{7}, 40 \mathrm{ng}$ of $26(27)$ hydroxycholesterol- $d_{5}, 80 \mathrm{ng}$ of 7-ketocholesterol- $d_{7}, 0.2 \mu \mathrm{g}$ of $5 \alpha$-cholestane, $0.2 \mu \mathrm{g}$ of desmosterol- $d_{6}, 0.2 \mu \mathrm{g}$ of lathosterol- $d_{4}, 0.2 \mu \mathrm{g}$ of campesterol- $d_{7}$ and $0.2 \mu \mathrm{g}$ of $\beta$-sitosterol- $d_{7}$ in $25 \mu \mathrm{l}$ of ethanol. Standards and sample mixtures were dried under a stream of $\mathrm{N}_{2}$ before adding the derivatizing agent $(15 \mu$ lacetonitrile and $15 \mu \mathrm{l}$ BSTFA + TMCS; Pierce Thermoscientific, Waltham, MA, USA). The derivatized samples were analyzed with an Agilent 5975 inert XL mass selective detector (Santa Clara, CA, USA). Selective ion monitoring was performed using the electron ionization mode at $70 \mathrm{eV}$ (with the ion source maintained at $230^{\circ} \mathrm{C}$ and the quadrupole at $150^{\circ} \mathrm{C}$ ) to monitor one target ion. Two qualifier ions were selected for the mass spectrum of each compound to optimize for sensitivity and specificity.

Ventral midbrain cultures. Ventral midbrains from 20 postnatal day 0 to day 2 rodents were dissected and digested in papain solution and plated on a glia layer. Cells were cultured in serum-free neurobasal/B27 medium (Invitrogen, Carlsbad, CA, USA) supplemented with superoxide dismutase $1(5 \mu \mathrm{g} / \mathrm{ml})$, apo-transferrin $(95 \mu \mathrm{g} / \mathrm{ml})$ and insulin $(21 \mu \mathrm{g} / \mathrm{ml})$ (all from Sigma). In contrast to Rayport et al., ${ }^{40}$ our 
culture medium contained no serum because the cultures were subjected to lipid addition in experimental conditions. One hour after plating the cells, $10 \mathrm{ng} / \mathrm{ml}$ of glialderived neurotrophic factor (Millipore, Billerica, MA, USA) was added. To inhibit glia growth, a solution of $16.5 \mathrm{mg} / \mathrm{ml}$ uridine and $6.7 \mathrm{mg} / \mathrm{ml} 5$-fluorodeoxyuridine was added 1 day after plating. Cells were then cultured for 7 days (days in vitro (DIV)7) before treatment with MPP + and various types of liposome.

Hippocampal neuron cultures. Hippocampal neurons from rats E18.5 embryos were cultured in neurobasal/B27 as described. ${ }^{19}$ Briefly, neurons were plated on a wax-dotted coverslip coated with $1 \mathrm{mg} / \mathrm{ml}$ of poly-D-lysine (Sigma). Two hours after plating, when neurons had attached, the coverslips were flipped into a six-well plate containing a glia feeder layer. PC, cholesterol or lanosterol liposome (5 $\mu \mathrm{m}$ each) was added after DIV7.

Western and antibodies. For western blots, cultured cells were washed three times with $1 \times$ PBS. Cells were lysed in $100 \mu$ l of RIPA buffer $(50 \mathrm{mM}$ Tris$\mathrm{HCl}, \mathrm{pH} 7.4,1 \% \mathrm{NP}-40,0.25 \%$ Na-deoxycholate, $150 \mathrm{mM} \mathrm{NaCl}$ and $1 \mathrm{mM}$ EDTA), supplemented with a cocktail of protease inhibitors (Complete Mini-EDTA inhibitors, Roche Diagnostics, Indianapolis, IN, USA). In all, $20 \mu \mathrm{g}$ of cell protein lysate was loaded in each well of a $10 \%$ polyacrylamide gel containing $0.1 \%$ SDS. After electrophoresis, proteins were transferred to nitrocellulose membrane and probed with the following antibodies: (i) rabbit anti-p35 (C19; 1:1000, Santa Cruz Biotechnology, Santa Cruz, CA, USA), (ii) rabbit anti-LSS (1:1000, AVIA, Systems Biology, San Diego, CA, USA), (iii) rabbit anti-tyrosine hydroxylase (TH) $(1: 20000$, Covance, Princeton, NJ, USA), (iv) rabbit anti-SREBP2 (1:1000, Abcam, Cambridge, MA, USA), (v) rabbit anti-calnexin (1:2000, Abcam), (vi) rabbit antiVDAC/porin (1:2000, Abcam) and (vii) rabbit anti-pGSK-3 $\alpha / \beta$ (ser21/9) $1: 1000$, Cell Signaling, Danvers, MA, USA). Peroxidase-conjugated anti-rabbit or antimouse secondary antibodies (1:10000) were purchased from Bio-Rad (Hercules, CA, USA). Immunoblots were visualized with enhance chemiluminescence reagent from Pierce Thermoscientific.

Fluorescence microscopy and quantification of dopaminergic neuronal survival. Ventral midbrain cultures plated on 12-mm coverslips were treated for $24 \mathrm{~h}$ with $10 \mu \mathrm{M} \mathrm{MPP}+$ with or without $5 \mu \mathrm{M} \mathrm{PC}, 5 \mu \mathrm{M}$ cholesterol or $5 \mu \mathrm{M}$ lanosterol liposome. Cells were washed three times with PBS, then fixed with $4 \%$ paraformaldehyde for 20 min, followed by permeabilization and blocking in $5 \%$ fetal bovine serum in $0.1 \%$ TritonX-100 for $30 \mathrm{~min}$. Cells were then stained with antiTH (secondary: Alexa-fluor 488, green) and neuron-specific class III beta tubulin (TUJ1) (secondary: Alexa-fluor 555, red). Anti-mouse and anti-rabbit Alexa-fluor 488 and 555 (1:1000) were purchase from Molecular Probes (Eugene, OR, USA)/ Invitrogen. TUJ1-positive and TH-positive cells were counted with an Olympus fluorescence microscope (Tokyo, Japan) with FITC and TRITC filter sets. Every neuron on the 12-mm coverslip was counted. The percentage of dopaminergic neurons in each group was determined by the number of TH + /TUJ1 + cells. Typically, in each control coverslip, there were 2000-3000 TUJ1 + cells, of which 400-1200 were $\mathrm{TH}+$. For each treatment, we assessed 4-6 coverslips per independent experiment. The averages of $4-5$ independent experiments are shown in the figures.

Confocal microscopy and colocalization studies. Ventral midbrain cultures in control and MPP +-treated cells were stained with rabbit anti-LSS (1:100), monoclonal mouse anti-TOMM20 (1:500) (Abcam) or monoclonal mouse anti-KDEL (1:500) (Abcam), and sheep anti-TH (1:500) (Abcam). The secondary antibodies, goat anti-mouse Alexa-fluor 555, goat anti-rabbit Alexa-fluor 488 and donkey anti-sheep Alexa-fluor 633, were obtained from Invitrogen. Cells were imaged with a laser-scanning confocal microscope (LSM510, Carl Zeiss, Oberkochen, Germany) with excitation and emission filters meeting the secondary Alexa-fluor antibody dye specifications. Images were taken using a $63 X$ objective. To quantify colocalization, we plotted the pixel intensities of LSS versus KDEL or TOMM20 from regions of interest (ROls) drawn around single neurons (either TH-positive or -negative), and calculated the linear regression coefficient, $R^{2}$, for $16-22$ individual $\mathrm{ROI} /$ neuron.

Live-cell confocal imaging and measurement of mitochondrial membrane potential. Mitochondrial membrane potential was measured in live neurons using $\mathrm{JC}-1$. All live-imaging experiments were conducted in cell medium, $\left(37^{\circ} \mathrm{C}\right.$ and $\left.5 \% \mathrm{CO}_{2}\right)$ with a spinning disk confocal microscope, equipped with a Cool SNAP HQ2 CCD camera (Photometrics, Tucson, AZ, USA), a fully automated stage and built in autofocusing system (PFS, Nikon, Tokyo, Japan) and driven by Metamorph 7.6 (Universal Imaging, Ypsilanti, MI, USA). JC-1 is excited at 488-nm, and its fluorescence emission was collected at $530 \pm 10 \mathrm{~nm}$ (green) and $590 \pm 17 \mathrm{~nm}$ (red), corresponding to peak fluorescence from the monomer and aggregate signals, respectively. Mitochondrial membrane potential was measured by taking the red to green emission ratio.

For ventral midbrain cultures (DIV7), cells were seeded at $50 \mathrm{cells} / \mathrm{mm}^{2}$ in a glass-bottom labtek well. Cells were loaded with $1 \mu \mathrm{g} / \mathrm{ml} \mathrm{JC}$ - 1 (Invitrogen) in culture medium, and were incubated for $30 \mathrm{~min}$ at $37^{\circ} \mathrm{C}$, washed twice with HBSS and imaged in conditioned culture medium. Multi-position time-lapse imaging of 10-15 randomly chosen fields was performed at 2-min interval over $40 \mathrm{~min}$. At the end of the experiment, ventral midbrain cells were fixed on stage for 20 min with $4 \%$ PFA and stained for $\mathrm{TH}$ with a secondary antibody coupled to Alexafluro-568 (red) and DAPI. The retrospective staining of TH allowed for the identification of dopaminergic neurons, which were the only ones included in the analyses. Hippocampal cultures were plated at a higher density $\left(300 \mathrm{cells} / \mathrm{mm}^{2}\right)$, and multi-position time-lapse imaging of 3-4 fields was performed at 30-s intervals for $20 \mathrm{~min}$.

For both types of cultures, the intensity of the JC-1 red-to-green ratio was measured in each frame, and the change in mitochondrial membrane potential was plotted as $\Delta \mathrm{f} / \mathrm{f}_{0}$, where $\mathrm{f}_{0}$ is the average $\mathrm{JC}$ - 1 red-to-green ratio over the first 10 frames before treatment. Decay curves were fitted to a mono-exponential function, $\mathrm{y}=\mathrm{x}_{0} \mathrm{e}^{-\mathrm{t} / \tau}$, using IGOR Pro 6.1 (WaveMetrics Inc., Lake Oswego, OR, USA).

Measurement of CoQ. For each condition, 3 to 4 million hippocampal neurons (DIV7) were treated with various lipids and incubated for $24 \mathrm{~h}$. Cells were washed two times with cold PBS and scraped in $0.5 \mathrm{ml}$ of cold PBS. Cells were centrifuged at $1000 \times g$ to pellet the cells, and resuspended in $100 \mu \mathrm{l}$ fresh PBS. Next, $750 \mu$ l of hexane/ethanol $(5: 2 \mathrm{v} / \mathrm{v})$ was added, and samples were vigorously vortexed for $1 \mathrm{~min}$. To extract $\mathrm{CoQ}, 400 \mu \mathrm{l}$ of the organic phase was collected and completely dried under a stream of $\mathrm{N}_{2}$, followed by LC-MS analysis.

An Agilent HPLC 1200 system coupled with an Applied Biosystems 3200 QTrap mass spectrometer (Foster City, CA, USA) was used for measuring CoQ8, Q9, and Q10 and free cholesterol. Chromatographic separation was carried out using an Agilent Zorbax Eclipse XDB-C18 column (i.d. $4.6 \times 150 \mathrm{~mm}$ ). High-pressure liquid chromatography (HPLC) conditions were as the following: mobile phase: chloroform/methanol $1: 1(\mathrm{v} / \mathrm{v})$; flow rate: $0.5 \mathrm{ml} / \mathrm{min}$; column temperature: $30^{\circ} \mathrm{C}$; injection volume: $10 \mu \mathrm{l}$. The LC-MS instrument was operated in positive atmospheric pressure chemical ionization mode with a vaporizer temperature of $500^{\circ} \mathrm{C}$ and a corona current of $3 \mu \mathrm{A}$. CoQ6 was used as an internal standard and monitored with an multiple reaction monitoring (MRM) transition of 591.4197.0. MRM transitions of 727.5197.0,795.5197.0 and 863.6197.0 were set up for analysis of CoQ8, Q9 and Q10, respectively.

Quantification of AVs. Ventral midbrain cultures plated on 12-mm coverslips were treated for $24 \mathrm{~h}$ with $10 \mu \mathrm{M}$ MPP + with or without $5 \mu \mathrm{M} \mathrm{PC}, 5 \mu \mathrm{M}$ cholesterol or $5 \mu \mathrm{M}$ lanosterol. Cells were washed three times with PBS, then fixed with $4 \%$ paraformaldehyde for $20 \mathrm{~min}$ and permeabilized with $100 \mu \mathrm{g} / \mathrm{ml}$ of digitonin for $10 \mathrm{~min}$. Following permeabilization, cells were washed three times with PBS and stained with anti-TH (secondary: Alexa-fluor 488, green) and anti-LC3 (1:100, mouse monoclonal, MBL cat. no.: 152-3A, secondary: Alexa-fluor 555, red). To quantify $\mathrm{AV}$ in dopaminergic neurons, $\mathrm{TH}+$ cell soma were imaged with a laserscanning confocal microscope (LSM510, Carl Zeiss) with excitation and emission filters meeting the secondary Alexa-fluor antibody dye specifications. Images were taken using a 63X objective with the same laser power and gain. The 12-bit images were quantified using ImageJ (analyze particle drop-in, National Institute of Health, Bethesda, MD, USA). For each image, detected LC3 puncta were intensity thresholded $(<1000)$ and gated for size $(<15$ pixel). For each condition, $40-60 \mathrm{TH}+$ cells were assessed from three independent experiments.

As a positive control for our method of evaluating AV, mouse embryonic fibroblast grown in serum or serum deprived were stained with LC3 and quantified according to the same parameters. As expected and shown in supplementary data (Supplementary Figure S2), there is approximately a 25 -fold increase in AV on serum starvation.

Quantification of mitophagy in axons. E18.5 hippocampal neurons were electroporated using the Amaxa poration system (Lonza, Basel, Switzerland) with MitoRed construct (Clonetech, Mountain View, CA, USA, cat. No.: PT-3633-5). Cells were then plated in microfluidic chambers (Xona Microfluidics, Temecula, CA, USA), which allow for physical separation of axons and cell somas. At DIV7, 
neurons were treated with $5 \mu \mathrm{M} \mathrm{PC}, 5 \mu \mathrm{M}$ cholesterol, $5 \mu \mathrm{M}$ lanosterol or $200 \mathrm{nM}$ of rapamycin (positive control) for $24 \mathrm{~h}$. Cells were then fixed with $4 \%$ paraformaldehyde for $20 \mathrm{~min}$ and permeabilized with $100 \mu \mathrm{g} / \mathrm{ml}$ of digitonin for $10 \mathrm{~min}$. Following permeabilization, cells were washed three times with PBS, and stained with anti-LC3 (secondary: Alexa-fluor 488, green). To quantify mitophagy, the percentage of MitoRed and LC3-positive mitochondria were plotted as a percentage to total MitoRed-positive mitochondria using the Metamorph 7.6 colocalization drop-in. For each condition, images of $40-50$ axons were taken from three independent experiments.

Statistical analyses. Error bars represent S.E.M. For fold changes in stero intermediates, $P$-values were calculated with two-tailed Mann-Whitney U-test. For in vitro assessment of dopaminergic neuron survival, CoQ levels, ATP levels, AV number and size, percentage of mitophagy, $P$-values were calculated with two-tailed Student's $t$-test.

\section{Conflict of Interest}

The authors declare no conflict of interest.

Acknowledgements. We thank Thilo Hagen for providing us with an aliquot of CCCP. This study was supported by the National University of Singapore (NUS), Department of Biological Sciences fellowship, the Singapore Biomedical Research Council (BMRC) Grant ID 08/1/21/19/558, the Singapore National Research Foundation under CRP Award No. 2007-04 and the SystemsX.ch RTD project LipidX.

1. Lesage S, Brice A. Parkinson's disease: from monogenic forms to genetic susceptibility factors. Hum Mol Genet 2009; 18 (R1): R48-R59.

2. Dauer W, Przedborski S. Parkinson's disease: mechanisms and models. Neuron 2003; 39 889-909.

3. Cookson MR. DJ-1, PINK1, and their effects on mitochondrial pathways. Mov Disord 2010; 25(Suppl 1): S44-S48.

4. Keeney PM, Xie J, Capaldi RA, Bennett Jr JP. Parkinson's disease brain mitochondria complex I has oxidatively damaged subunits and is functionally impaired and misassembled. J Neurosci 2006; 26: 5256-5264.

5. Liu Y, Yang H. Environmental toxins and alpha-synuclein in Parkinson's disease. $\mathrm{Mol}$ Neurobiol 2005; 31: 273-282.

6. Virmani A, Gaetani F, Binienda Z. Effects of metabolic modifiers such as carnitines, coenzyme Q10, and PUFAs against different forms of neurotoxic insults: metabolic inhibitors, MPTP, and methamphetamine. Ann NY Acad Sci 2005; 1053 183-191.

7. Horvath $T L$, Diano S, Leranth C, Garcia-Segura LM, Cowley MA, Shanabrough M et al. Coenzyme $Q$ induces nigral mitochondrial uncoupling and prevents dopamine cell loss in a primate model of Parkinson's disease. Endocrinology 2003; 144: 2757-2760.

8. Andrews ZB, Horvath B, Barnstable CJ, Elsworth J, Yang L, Beal MF et al. Uncoupling protein-2 is critical for nigral dopamine cell survival in a mouse model of Parkinson's disease. J Neurosci 2005; 25: 184-191.

9. Conti B, Sugama S, Lucero J, Winsky-Sommerer R, Wirz SA, Maher P et al. Uncoupling protein 2 protects dopaminergic neurons from acute 1,2,3,6-methyl-phenyltetrahydropyridine toxicity. J Neurochem 2005; 93: 493-501.

10. Guzman JN, Sanchez-Padilla J, Wokosin D, Kondapalli J, lijic E, Schumacker PT et al. Oxidant stress evoked by pace making in dopaminergic neurons is attenuated by DJ-1. Nature 2010; 468: 696-700.

11. Stout AK, Raphael HM, Kanterewicz BI, Klann E, Reynolds IJ. Glutamate-induced neuron death requires mitochondrial calcium uptake. Nat Neurosci 1998; 1: 366-373.

12. Huang $X$, Chen $\mathrm{H}$, Miller WC, Mailman RB, Woodard JL, Chen PC et al. Lower low-density lipoprotein cholesterol levels are associated with Parkinson's disease. Mov Disord 2007; 22: 377-381.

13. de Lau LM, Koudstaal PJ, Hofman A, Breteler MM. Serum cholesterol levels and the risk of Parkinson's disease. Am J Epidemiol 2006; 164: 998-1002.

14. Broersen K, Brink D, Fraser G, Goedert M, Davletov B. Alpha-synuclein adopts an alphahelical conformation in the presence of polyunsaturated fatty acids to hinder Micelle formation. Biochemistry 2006; 45: 15610-15616.

15. Bosco DA, Fowler DM, Zhang Q, Nieva J, Powers ET, Wentworth Jr P et al. Elevated levels of oxidized cholesterol metabolites in Lewy body disease brains accelerate alpha-synuclein fibrilization. Nat Chem Biol 2006; 2: 249-253.

16. Andreyev AY, Fahy E, Guan Z, Kelly S, Li X, McDonald JG et al. Subcellular organelle lipidomics in TLR-4-activated macrophages. J Lipid Res 2010; 51: 2785-2797.

17. Jackson-Lewis V, Jakowec M, Burke RE, Przedborski S. Time course and morphology of dopaminergic neuronal death caused by the neurotoxin 1-methyl-4-phenyl-1,2,3,6tetrahydropyridine. Neurodegeneration 1995; 4: 257-269.

18. Vance JE, Hayashi $\mathrm{H}$, Karten B. Cholesterol homeostasis in neurons and glial cells. Semin Cell Dev Biol 2005; 16: 193-212.

19. Kaech S, Banker G. Culturing hippocampal neurons. Nat Protoc 2006; 1: 2406-2415.

20. Fernandez A, Llacuna L, Fernandez-Checa JC, Colell A. Mitochondrial cholesterol loading exacerbates amyloid beta peptide-induced inflammation and neurotoxicity. I Neurosci 2009; 29: 6394-6405

21. Neystat M, Rzhetskaya M, Oo TF, Kholodilov N, Yarygina O, Wilson A et al. Expression of cyclin-dependent kinase 5 and its activator p35 in models of induced apoptotic death in neurons of the substantia nigra in vivo. J Neurochem 2001; 77: 1611-1625.

22. Garcia-Gorostiaga I, Sanchez-Juan P, Mateo I, Rodriguez-Rodriguez E, SanchezQuintana C, del Olmo SC et al. Glycogen synthase kinase-3 beta and tau genes interact in Parkinson's and Alzheimer's diseases. Ann Neurol 2009; 65: 759-761 author reply 761-752.

23. Mori M, Sawashita J, Higuchi K. Functional polymorphisms of the LSS and Fdtt1 genes in laboratory rats. Exp Anim 2007; 56: 93-101.

24. Yamamoto S, Lin K, Bloch K. Some properties of the microsomal 2,3-oxidosqualene sterol cyclase. Proc Natl Acad Sci U S A 1969; 63: 110-117.

25. Ramsay RR, Singer TP. Energy-dependent uptake of N-methyl-4-phenylpyridinium, the neurotoxic metabolite of 1-methyl-4-phenyl-1,2,3,6-tetrahydropyridine, by mitochondria. J Biologic Chem 1986; 261: 7585-7587.

26. Narendra D, Tanaka A, Suen DF, Youle RJ. Parkin is recruited selectively to impaired mitochondria and promotes their autophagy. J Cell Biol 2008; 183: 795-803.

27. Matsuda N, Sato S, Shiba K, Okatsu K, Saisho K, Gautier CA et al. PINK1 stabilized by mitochondrial depolarization recruits Parkin to damaged mitochondria and activates latent Parkin for mitophagy. J Cell Biol 2010; 189: 211-221.

28. Geisler S, Holmstrom KM, Treis A, Skujat D, Weber SS, Fiesel FC et al. The PINK1/Parkinmediated mitophagy is compromised by PD-associated mutations. Autophagy 2010; 6 : 871-878.

29. Cherra 3rd SJ, Kulich SM, Uechi G, Balasubramani M, Mountzouris J, Day BW et al. Regulation of the autophagy protein LC3 by phosphorylation. J Cell Biol 2010; 190: 533-539.

30. Park JW, Kim HJ, Byun JH, Ryu HR, Jeon NL. Novel microfluidic platform for culturing neurons: culturing and biochemical analysis of neuronal components. Biotechnol J 2009; 4 : 1573-1577.

31. Haas RH, Nasirian F, Nakano K, Ward D, Pay M, Hill R et al. Low platelet mitochondrial complex I and complex II/II activity in early untreated Parkinson's disease. Ann Neurol 1995; 37: 714-722.

32. Huang X, Abbott RD, Petrovitch H, Mailman RB, Ross GW. Low LDL cholesterol and increased risk of Parkinson's disease: prospective results from Honolulu-Asia Aging Study. Mov Disord 2008; 23: 1013-1018.

33. Becker C, Meier CR. Statins and the risk of Parkinson disease: an update on the controversy. Expert Opin Drug Saf 2009; 8: 261-271.

34. Kolsch H, Heun R, Jessen F, Popp J, Hentschel F, Maier W et al. Alterations of cholestero precursor levels in Alzheimer's disease. Biochimica et biophysica acta 2011; 1801: 945-950.

35. Blanc M, Hsieh WY, Robertson KA, Watterson S, Shui G, Lacaze P et al. Host defense against viral infection involves interferon mediated down-regulation of sterol biosynthesis. PLOS Biol 2011; 9: e1000598.

36. Brennan JP, Southworth R, Medina RA, Davidson SM, Duchen MR, Shattock MJ. Mitochondrial uncoupling, with low concentration FCCP, induces ROS-dependent cardioprotection independent of KATP channel activation. Cardiovasc Res 2006; 72: 313-321.

37. Geisler S, Holmstrom KM, Skujat D, Fiesel FC, Rothfuss OC, Kahle PJ et al. PINK1/Parkinmediated mitophagy is dependent on VDAC1 and p62/SQSTM1. Nat Cell Biol 2010; 12 . $119-131$

38. Okatsu K, Saisho K, Shimanuki M, Nakada K, Shitara H, Sou YS et al. p62/SQSTM1 cooperates with Parkin for perinuclear clustering of depolarized mitochondria. Genes Cells 2010; 15: 887-900.

39. Bligh EG, Dyer WJ. A rapid method of total lipid extraction and purification. Can J Biochem Physiol 1959; 37: 911-917.

40. Rayport S, Sulzer D, Shi WX, Sawasdikosol S, Monaco J, Batson D et al. Identified postnatal mesolimbic dopamine neurons in culture: morphology and electrophysiology. J Neurosci 1992; 12: 4264-4280.

(c)

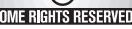
Unported License. To view a copy of this license, visit http:// creativecommons.org/licenses/by-nc-nd/3.0

\section{Supplementary Information accompanies the paper on Cell Death and Differentiation website (http://www.nature.com/cdd)}

